\title{
Supporting information for: \\ Genetically encoded split-luciferase biosensors to measure endosome disruption rapidly in live cells
}

Kameron V. Kilchrist, John William Tierney, and Craig L. Duvall*

Department of Biomedical Engineering, Vanderbilt University, Nashville, TN, USA.

*Corresponding author email address: craig.duvall@vanderbilt.edu

Contents

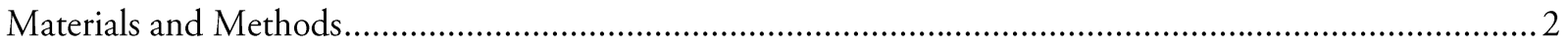

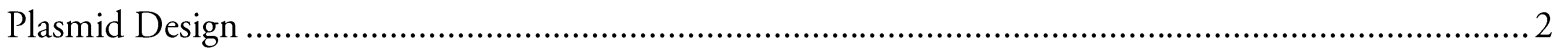

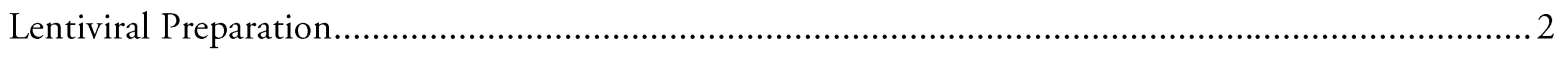

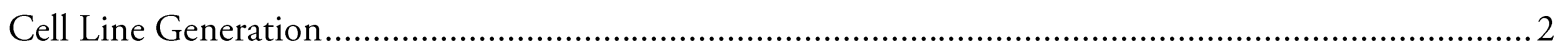

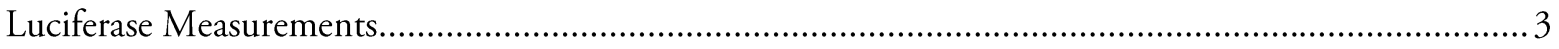

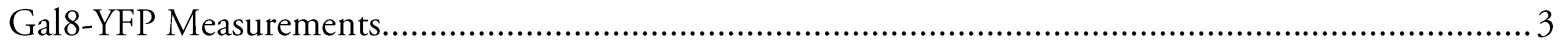

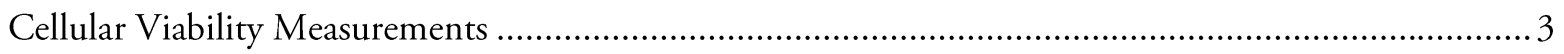

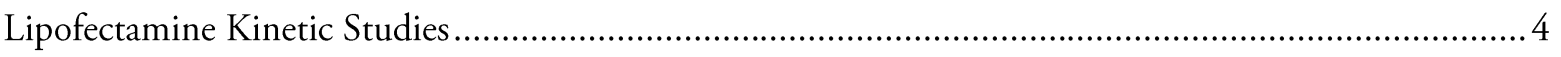

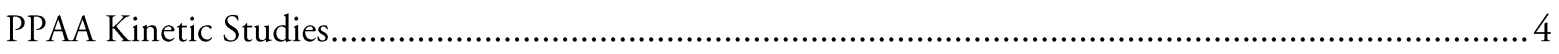

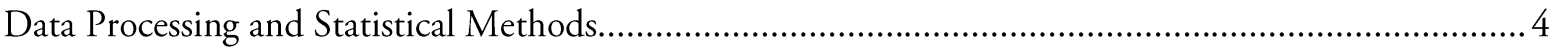

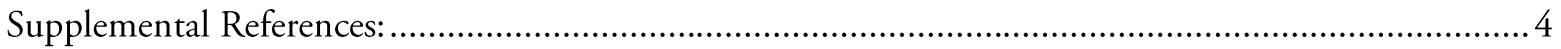

Figure S1. Graphical overview of the G8C2 and G8G8 transcripts.................................................. 5

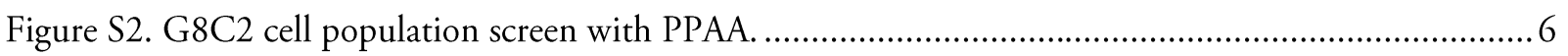

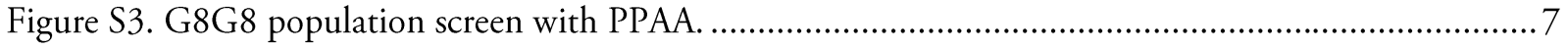

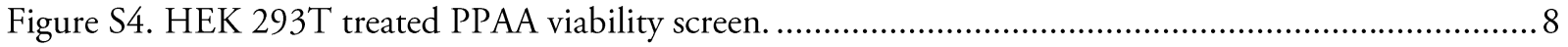

Figure S5. HEK 293T cell line establishment Gal8-YFP dataset......................................................... 9

Figure S6. Previously reported 50B polymer bioactivity (gene knockdown) and Gal8-YFP recruitment data for polymers with varied molecular weight. ................................................................................. 10

Figure S7. DMSO and ethanol are not sufficient to induce G8G8 response......................................... 11

Figure S8. Lipofectamine 2000 time course of G8G8 split-luciferase signal......................................... 12

Figure S9. Timepoint testing of PPAA with G8G8 assay system........................................................ 13

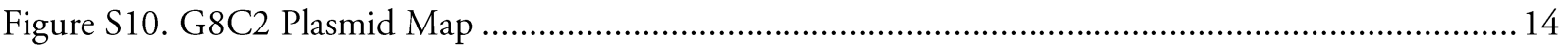

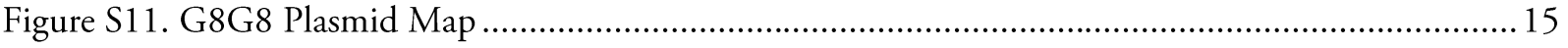




\section{Materials and Methods}

\section{Plasmid Design}

Lentiviral transfer plasmids were designed in silico and generated by VectorBuilder (Chicago, IL, USA). The G8C2 system was designed as follows: the first ORF contained a human eukaryotic translation elongation factor 1 alphal short form (EFS) promoter driving an mRNA transcript containing NLuc398 (Luciferase AA 1-398), a "GGGGS" triplet spacer (3xG4S), and full-length Gal8 (hLGALS8, NM_201545.2), followed by full-length human CALCOCO2 (NM_001261390.1), 3xG4S, CLuc394 (Luciferase AA 394-550), then a stop codon. The second ORF consisted of a cytomegalovirus (CMV) promoter driving blasticidin-S deaminase. The G8C2 plasmid has been deposited with AddGene.com (ID 128387). A plasmid map is shown as Figure S11.

The G8G8 system was designed as follows: the first ORF contained a human eukaryotic translation elongation factor 1 alpha1 (EF1A) promoter driving an mRNA transcript containing NLuc398 in frame with a 3xG4S spacer to the first 157 amino acids of human Galectin 8, corresponding to the $\mathrm{N}$-terminal carbohydrate recognition domain (G8-NCRD) with a stop codon, followed by an internal ribosome entry site sequence derived from the encephalomyocarditis virus upstream of CLuc394, a 3xG4S spacer, followed by the same G8NCRD. The second ORF consisted of a cytomegalovirus (CMV) promoter driving enhanced green protein connected to blasticidin-S deaminase via a T2A self-cleaving peptide. The G8G8 plasmid has been deposited with AddGene.com (ID 128388). A plasmid map is shown as Figure S12.

The NLuc and CLuc degree of luciferase fragment overlap (4 AA) and split point (1 - 398; 394 - 550)

were chosen based on work by Paulmurugan and Gambhir, ${ }^{1}$ which showed this design to produce strong luminescence upon protein-protein interaction.

\section{Lentiviral Preparation}

Pseudotyped lentiviral particles (LV) were generated using transfer plasmids (above), pCMV delta R8.2 (AddGene Cat. No. 12263), and pMD2.G (AddGene Cat. No. 12259). Such that a final volume of $600 \mu \mathrm{L}$ was reached, the following components were added, in order, to a $15 \mathrm{~mL}$ polypropylene conical tube to yield a transfection mixture: Opti-MEM media (Gibco, Cat. No. 31985062), $0.6 \mu \mathrm{g}$ pMD2.G, $3.0 \mu \mathrm{g}$ pCMV delta R8.2, $6.0 \mu \mathrm{g}$ transfer plasmids, and $42 \mu \mathrm{L}$ FuGENE 6 (Promega, Cat. No. E2691). The tube was gently flicked to mix the plasmids before and after the addition of FuGENE 6. The transfection mixture was added dropwise to a T-75 flask at approximately 50\% confluency of HEK 293T (American Type Culture Collection Cat. No. CRL-3216) cells in $11.0 \mathrm{~mL}$ DMEM/F-12 supplemented to $10 \%$ FBS without antibiotics. After $18 \mathrm{~h}$ incubation, the media on the HEK 293T cells was exchanged for DMEM supplemented with 10\% FBS and $1 \%$ penicillin-streptomycin. At $24 \mathrm{~h}, 48 \mathrm{~h}$, and $72 \mathrm{~h}$ after this media change, the virus-containing supernatant was harvested, syringe filtered $(0.45 \mu \mathrm{m}$, nylon), and concentrated from $-11 \mathrm{~mL}$ to $-250 \mu \mathrm{L}$ with Amicon Ultra-15 centrifugal filtration unit (100 kDa nominal molecular weight cut off). The LV were then either used immediately or aliquoted and frozen for later use.

\section{Cell Line Generation}

A 2-fold dilution series of LV was made in serum free, HEPES supplemented DMEM, starting with undiluted viral concentrate $\left(2^{0}\right)$ and ending with $2^{-11}$ viral supernatant. To 12 wells of a 96-well plate, $100 \mu \mathrm{L}$ of $10^{5}$ cells $/ \mathrm{mL}$ were added. Then, $100 \mu \mathrm{L}$ from the dilution series was added to the plate of cells. After $48 \mathrm{~h}$ in which the cells adhered and proliferated, the media was changed and selection began, using DMEM supplemented with $10 \%$ FBS and $5 \mu \mathrm{g} / \mathrm{mL}$ blasticidin. Cells were monitored by phase contrast microscopy; as wells reached confluence, cells were washed twice with PBS -/-, trypsinized with a minimal volume of $0.25 \%$ trypsin, diluted with serum containing media, and transferred without centrifugation to progressively larger 
vessels. Media was changed every $48-72 \mathrm{~h}$. As each T-75 flask reached $-80 \%$ confluence, half the cells were aliquoted and stored cryogenically in a 90:10 v/v mixture of FBS and DMSO and further expanded using T175 plates. Cells were periodically monitored throughout this process for EGFP expression via microscopy or for luciferase expression by IVIS imaging. HEK 293T cells stably expressing G8G8 or G8C2 are available upon request from the corresponding author. Cells originating from the first five dilutions were denoted Population 1 through Population 5 and used herein. Cells were maintained on blasticidin until the passage before plating for assays.

\section{Luciferase Measurements}

Cells were seeded at a concentration of 20,000 cells per well in $100 \mu \mathrm{L}$ media (Gibco DMEM supplemented with 10\% FBS and $25 \mathrm{mM} \mathrm{HEPES)}$ and allowed to grow to confluence. Luciferase measurements were taken on live cells by exchanging media with media supplemented with $10 \%$ FBS, 150 $\mu \mathrm{g} / \mathrm{mL}$ D-luciferin, and $25 \mathrm{mM}$ HEPES. Cells were imaged after 3-5 minutes of incubation at $37^{\circ} \mathrm{C}$ in clear bottom, black wall, 96-well plates in an IVIS Lumina Imaging system (Xenogen Corporation, Alameda, CA, USA) with $60 \mathrm{~s}$ exposure. Luminescence was monitored for up to $20 \mathrm{~min}$. Regions of interest were drawn using the well plate feature and average photon flux was calculated and exported. The data for Figure 2, S2, and S3 represent the results from a study with 3 replicates per dose. In the study, the wells along the edge of the plate were unused to avoid edge effects. Each dilution series was plated across a single row, and alternating rows of test article and buffer control were used to confirm that there was not variance due to position on the plate. The data shown are from a study that was representative of three independent runs of the experiment. For Figure 4D, one well of each dose/polymer combination was applied per plate across three plates to yield 3 technical replicates. The signal for each well was normalized to a paired vehicle control included on the same plate. Each polymer condition was plotted as the average of the \% paired control across the 3 plates, and error bars represent standard deviation.

\section{Gal8-YFP Measurements}

Gal8-YFP measurements were conducted as previously reported ${ }^{2}$ with two differences. First, HEK 293T were used instead of MDA-MB-231 to generate stable Gal8-YFP expressing cells. Second, recruited Gal8YFP was normalized to total cellular area rather than cell number by quantifying cellular area by thresholding pixels above background. Cells were seeded at 1,000 cells per well in $100 \mu \mathrm{L}$ and allowed to grow to $-50 \%$ confluency. HEK 293T Gal8-YFP cells and Gal8-YFP retrovirus concentrates are available upon request from the corresponding author. A PiggyBac compatible Gal8-GFP has also been made available by the Jordan Green lab at AddGene (ID 127191). ${ }^{3}$

\section{Cellular Viability Measurements}

Cells were seeded at a concentration of 20,000 cells per well in $100 \mu \mathrm{L}$ media (Gibco DMEM supplemented with 10\% FBS and $25 \mathrm{mM} \mathrm{HEPES)}$ and allowed to grow to confluence into a 96-well plate. Cells were then treated with a 2-fold dilution series of PPAA, starting with $625 \mathrm{ug} / \mathrm{mL}$. After 2 hours, the PPAA media was removed and cells were washed once with DMEM supplemented with $10 \%$ FBS. Then, media was changed to DMEM supplemented to $10 \%$ FBS and $25 \mathrm{ug} / \mathrm{mL}$ resazurin salt. Plate absorbance was read on a TECAN Infinite M1000 Pro plate reader in a continuous kinetic cycle. 


\section{Lipofectamine Kinetic Studies}

HEK 293T G8G8 cells were seeded at a concentration of 20,000 cells per well in $100 \mu \mathrm{L}$ media (Gibco DMEM supplemented with $10 \%$ FBS and $25 \mathrm{mM} \mathrm{HEPES}$ ) and allowed to grow to confluence into a 96 well plate and were treated with $0.5 \% \mathrm{v} / \mathrm{v}$ Lipofectamine 2000 in serum free DMEM at 2, 8, or 24 hours before reading. After the specified amount of time, the media was replaced with luciferin-containing media and luminescence was measured on the IVIS.

\section{PPAA Kinetic Studies}

HEK 293T G8G8 cells were seeded at a concentration of 20,000 cells per well in $100 \mu \mathrm{L}$ media (Gibco DMEM supplemented with $10 \%$ FBS and $25 \mathrm{mM} \mathrm{HEPES}$ ) and allowed to grow to confluence into a 96 well plate and were treated with a 2 -fold dilution series of PPAA in serum-free DMEM starting with $625 \mathrm{ug} / \mathrm{mL}$. The PPAA treatment was left on the cells for 15, 30, 60, or 120 minutes, after which the media was replaced with luciferincontaining media and luminescence was measured on the IVIS.

\section{Data Processing and Statistical Methods}

Plots, regressions, and statistics were generated using GraphPad Prism 8. For the G8C2, G8G8, and Gal8-YFP systems, PPAA versus vehicle dose response curves were analyzed using ordinary two-way ANOVA with the following settings: no sample matching, column factor of putative endosome-disrupting agent versus buffer, row factor of dose, with post-hoc testing to compare dose-matched response of agent versus buffer. Sidak's multiple comparison correction was used to calculate a multiplicity-adjusted $\mathrm{P}$ value. The family-wise significance and confidence rate was set to 0.05 , or a $95 \%$ confidence interval. For figure S8, ordinary one-way ANOVA was performed with multiple comparisons testing against control. Throughout the manuscript, asterisks are used per the GraphPad Prism style: ${ }^{*}$ for $\mathrm{p}<0.05,{ }^{* *}$ for $\mathrm{p}<0.01,{ }^{* * *}$ for $\mathrm{p}<0.001$, and ${ }^{* * *}$ for $\mathrm{p}<0.0001$.

\section{Supplemental References:}

(1) Paulmurugan, R.; Gambhir, S. S. Combinatorial Library Screening for Developing an Improved SplitFirefly Luciferase Fragment-Assisted Complementation System for Studying Protein-Protein Interactions. Anal. Chem. 2007, 79 (6), 2346-2353.

(2) Kilchrist, K. V.; Dimobi, S. C.; Jackson, M. A.; Evans, B. C.; Werfel, T. A.; Dailing, E. A.; Bedingfield, S. K.; Kelly, I. B.; Duvall, C. L. Gal8 Visualization of Endosome Disruption Predicts Carrier-Mediated Biologic Drug Intracellular Bioavailability. ACS Nano 2019, acsnano.8b05482.

(3) Rui, Y.; Wilson, D. R.; Choi, J.; Varanasi, M.; Sanders, K.; Karlsson, J.; Lim, M.; Green, J. J. Carboxylated Branched Poly( $\beta$-Amino Ester) Nanoparticles Enable Robust Cytosolic Protein Delivery and CRISPR-Cas9 Gene Editing. Sci. Adv. 2019, 5 (12), eaay3255. 
Figure S1. Graphical overview of the G8C2 and G8G8 transcripts.

A. Gal8 / CALCOCO2 Interaction System (G8C2)

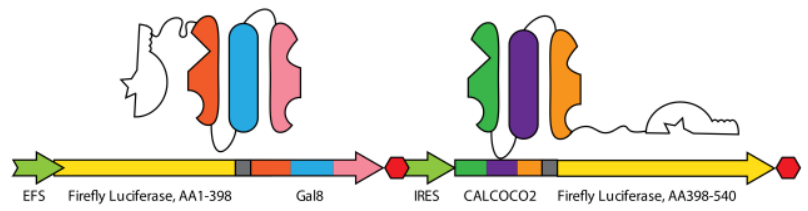

B. Gal8-NCRD / Gal8-NCRD Interaction System (G8G8)

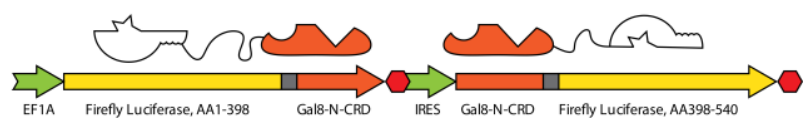

A. From a single mRNA transcript driven by the EFS promoter, two proteins are expressed. The first is NLuc-GGGGSx3-Gal8 and the second is CALCOCO2-GGGGSx3-CLuc, connected by an internal ribosomal entry site sequence. DNA and protein constructs are not to scale.

B. From a single mRNA transcript driven by the EF1A promoter, two proteins are expressed. The first is NLuc-GGGGSx3-G8-NCRD and the second is G8-NCRD-GGGGSx3-CLuc, connected by an internal ribosomal entry site sequence. DNA and protein constructs are not to scale. 
Figure S2. G8C2 cell population screen with PPAA.

A

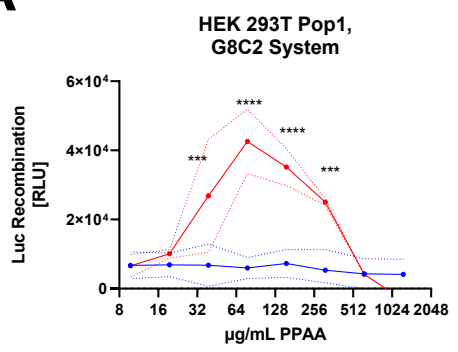

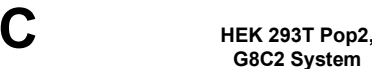

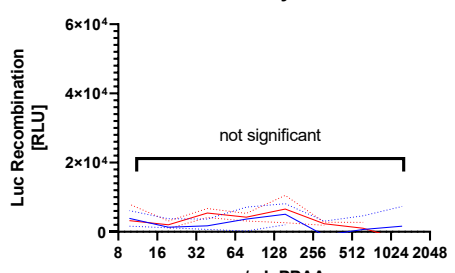

$\mu \mathrm{g} / \mathrm{mL}$ PPAA

E

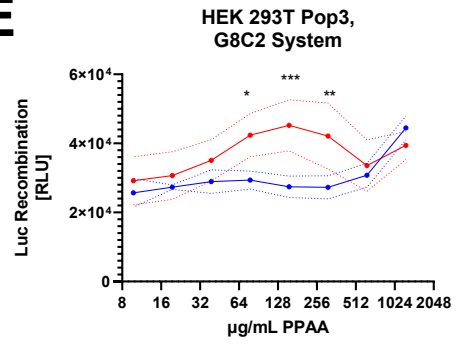

G

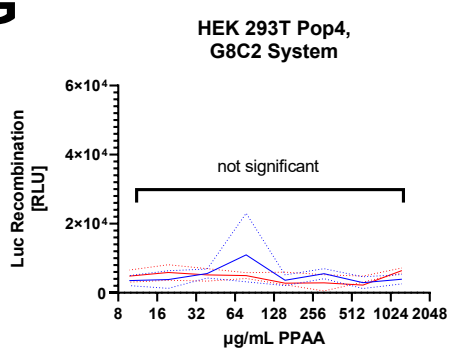

I

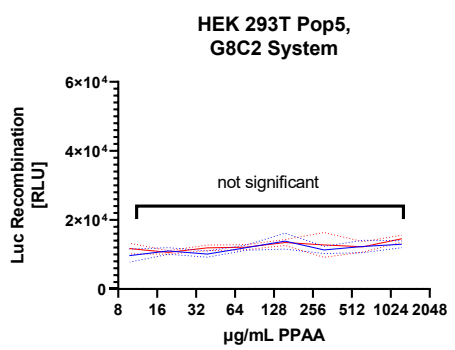

B

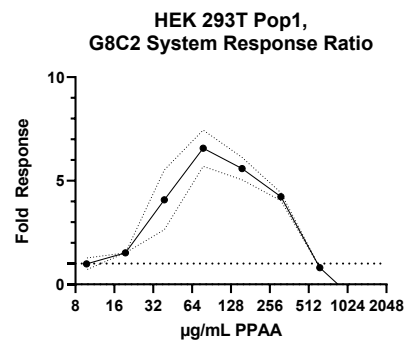

D

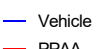

- PPAA

- Vehicle
— PPAA

$\mathbf{F}$

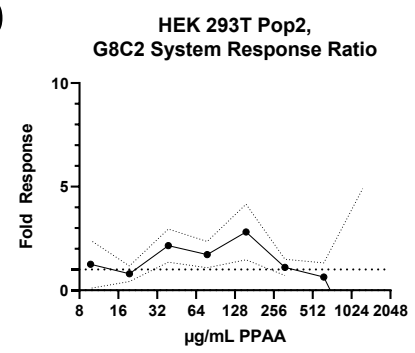

HEK 293T Pop3,

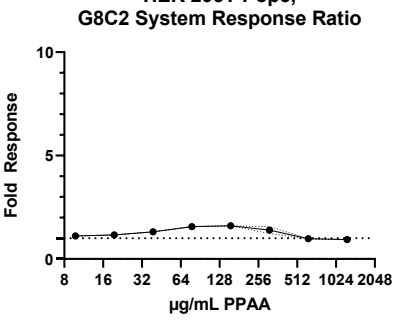

H $\mu \mathrm{g} / \mathrm{mL}$ PPAA

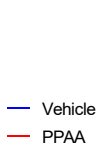

- VPAA

HEK 2937 Pop4 G8C2 System Response Ratio

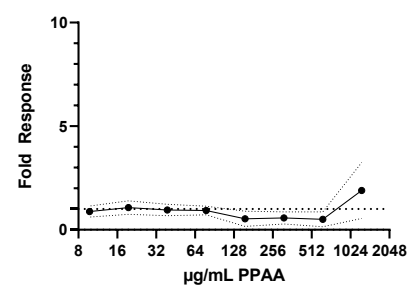

J

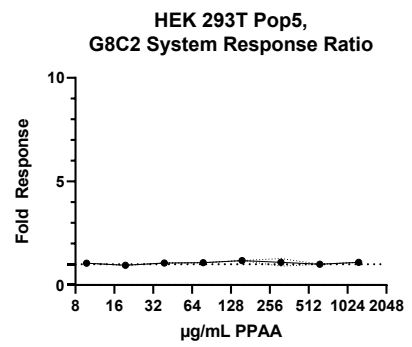

A, C, E, G, I: G8C2 cells were treated with a 2-fold serial dilution of PPAA for $2 \mathrm{~h}$, media exchanged, and luminescence measured. $\mathrm{p}$ values are indicated as: $\mathrm{p}<0.05,{ }^{*} ;<0.01^{* *} ;<0.001^{* * *} ;<0.0001^{* * * *}$

$\mathrm{B}, \mathrm{D}, \mathrm{F}, \mathrm{H}, \mathrm{J}$ : luminescent response was normalized to vehicle values and plotted as fold response versus PPAA concentration. A dotted line at $\mathrm{y}=1$ represents the average of buffer treated samples. Dashed lines represent standard deviation. Note that $\mathrm{S} 2$ and $\mathrm{S} 3$ do not utilize the same y-axis scale. 
Figure S3. G8G8 population screen with PPAA.

A

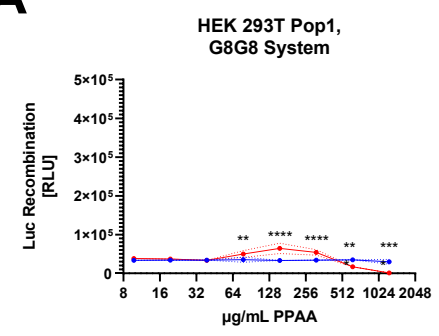

C

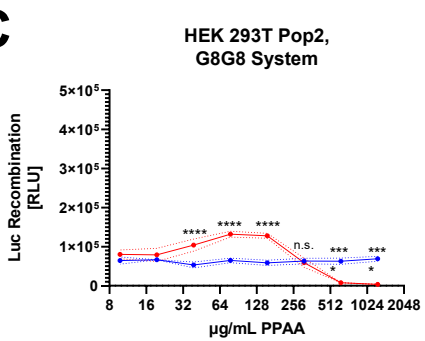

E

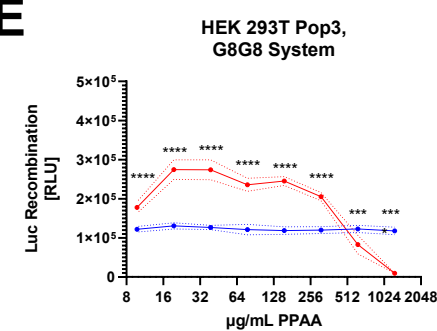

G

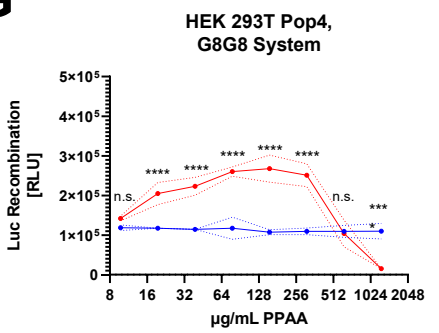

I

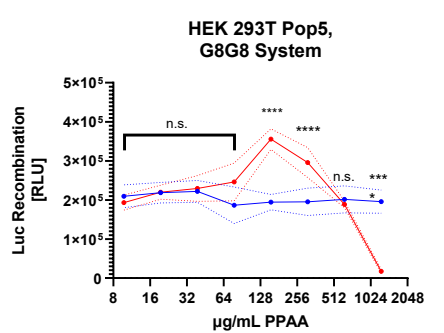

B

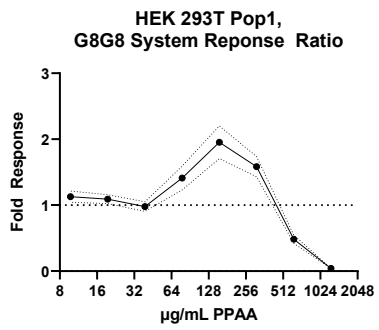

DEK 293T Pop2,

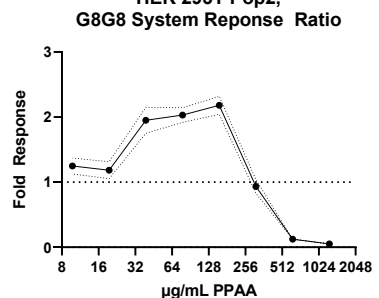

F HEK 2937 Pop3,

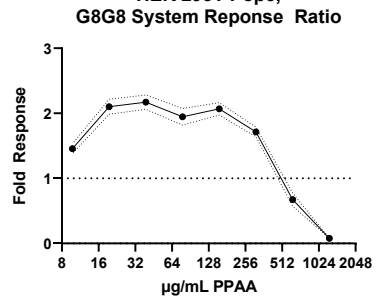

H

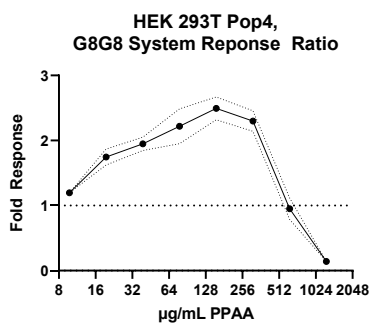

HEK 293T Pop5,

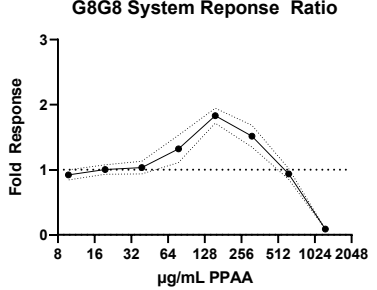

A, C, E, G, I: G8G8 cells were treated with a 2-fold serial dilution of PPAA for $2 \mathrm{~h}$, media exchanged, and luminescence measured. p values are indicated as: $\mathrm{p}<0.05{ }^{*} ;<0.01^{* *} ;<0.001^{* * *} ;<0.0001^{* * * *}$

$\mathrm{B}, \mathrm{D}, \mathrm{F}, \mathrm{H}$, J: luminescent response was normalized to vehicle values and plotted as fold response versus PPAA concentration. A dotted line at $\mathrm{y}=1$ represents the average of buffer treated samples. Dashed lines represent standard deviation. Note that $S 2$ and $S 3$ do not utilize the same $y$-axis scale. 
Figure S4. HEK 293T treated PPAA viability screen.

\section{Viability by Resazurin Assay}

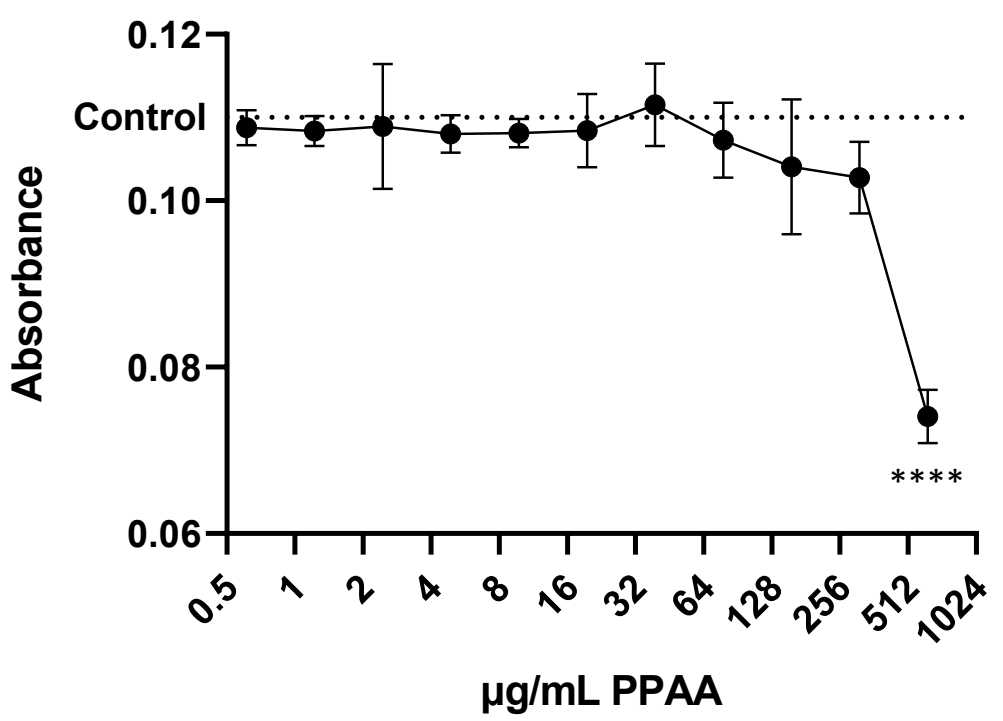

G8G8 HEK 293T cells were treated with a 2-fold dilution series from 625 to $0.6 \mu \mathrm{g} / \mathrm{mL}$ PPAA for $2 \mathrm{~h}$ or buffer control. Viability was assessed with the resazurin reduction assay. A notable loss of viability is observable at the highest dose. The average no treatment control signal is plotted as a dotted line. Error bars represent standard deviation. $\mathrm{p}$ values are indicated as: $\mathrm{p}<0.0001^{* * * *}$ as calculated by 1 -way ANOVA with Dunnett's multiple comparisons test against buffer control. 
Figure S5. HEK 293T cell line establishment Gal8-YFP dataset.

A
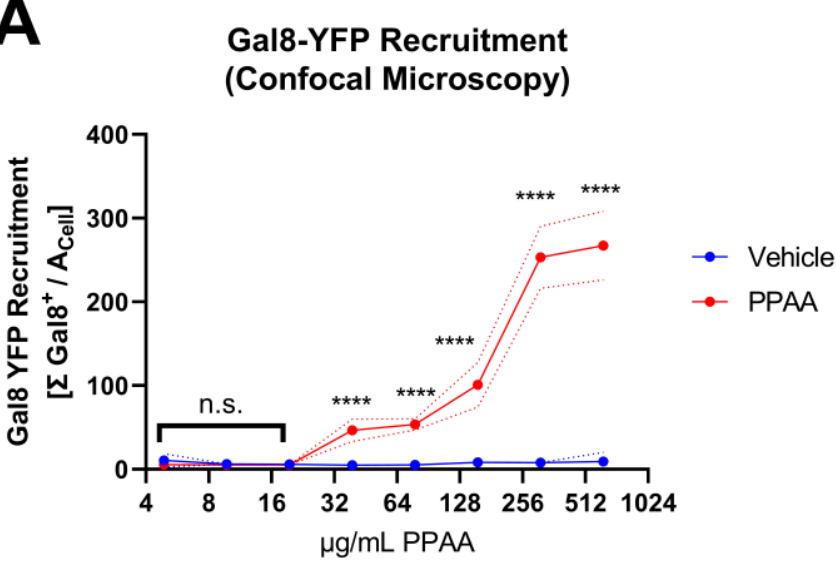

B

HEK293-T Gal8-YFP Response

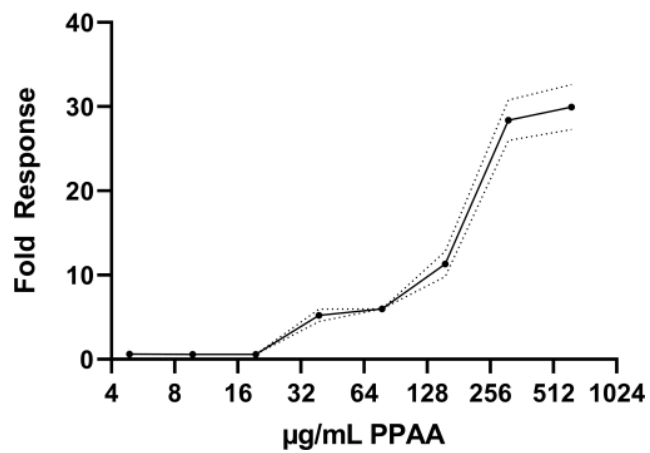

Gal8-YFP cells were treated with a 2-fold dilution series from 1250 to $9.8 \mu \mathrm{g} / \mathrm{mL}$ PPAA for $2 \mathrm{~h}$.

A. Gal8-YFP response data are plotted in red and buffer vehicle treated cells are plotted in blue. Significant differences were observed at the five highest doses. Dashed lines represent standard deviation.

B. The Gal8 response was normalized to the average buffer response and plotted as fold response. Dashed lines represent standard deviation. 
Figure S6. Previously reported 50B polymer bioactivity (gene knockdown) and Gal8-YFP recruitment data for polymers with varied molecular weight.

A. 50B MW Library, Luc Knockdown

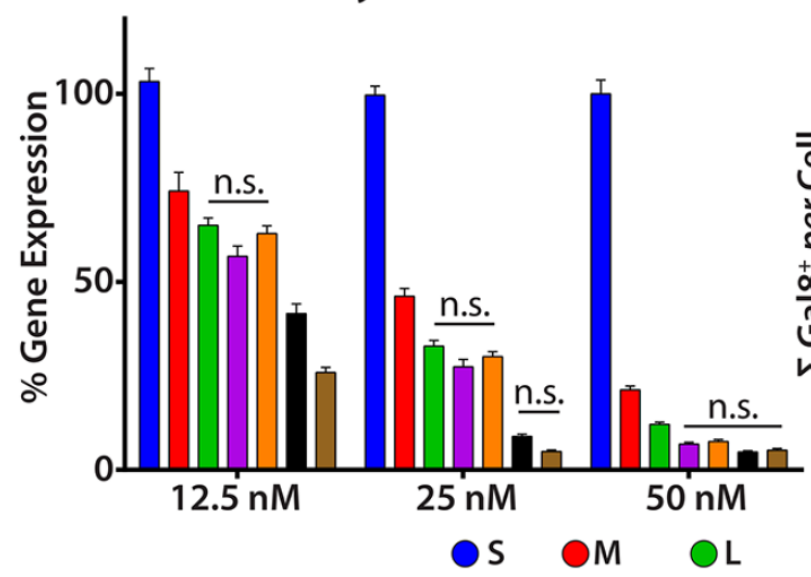

B. 50B MW Library, Gal8 Recruitment

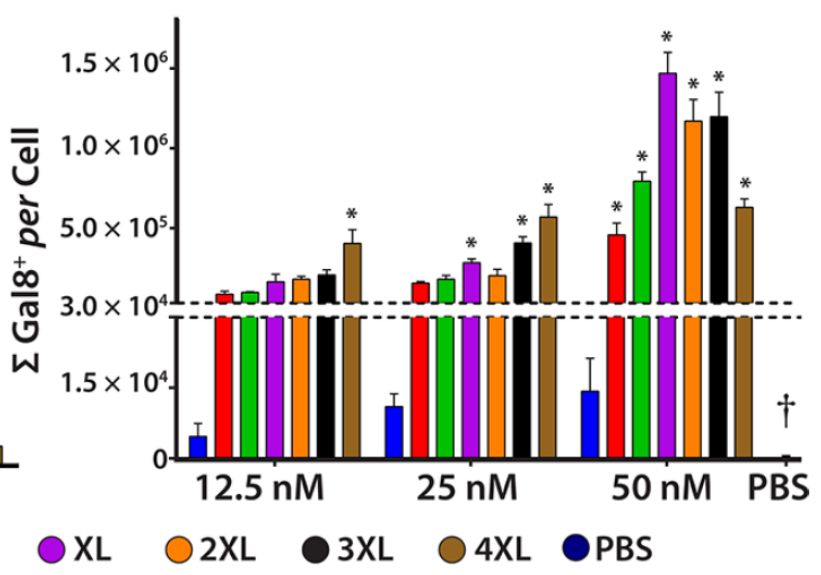

A. 50B shows dose-dependent anti-luciferase siRNA-mediated gene knockdown for 50B-M, L, XL, 2XL, 3XL, and 4XL, but not 50B-S polymers. Enhanced siRNA delivery is mediated by enhanced endosome disruption and results in reduced luciferase expression.

B. MDA-MB-231 Gal8-YFP cells were treated with noted polymers. Study revealed that Gal8 recruitment increases with increasing siRNA dose and increasing polymer MW. PBS-treated cells had near-zero response, highlighted with a dagger. Single asterisks indicate polymers identified as significant hits by Dunnet's comparison test.

These data demonstrate that 50B-S does not induce endosomal disruption, while 50B-L, 2XL, and 4XL, used in Figure 3D, cause significant endosomal disruption.

Reprinted with minor modifications for clarity with permission from our recent paper, Kilchrist et al., ACS Nano 2019, 13, 1136-1152. https://pubs.acs.org/doi/abs/10.1021/acsnano.8b05482

Copyright 2019 American Chemical Society. 
Figure S7. DMSO and ethanol are not sufficient to induce G8G8 response.

A.

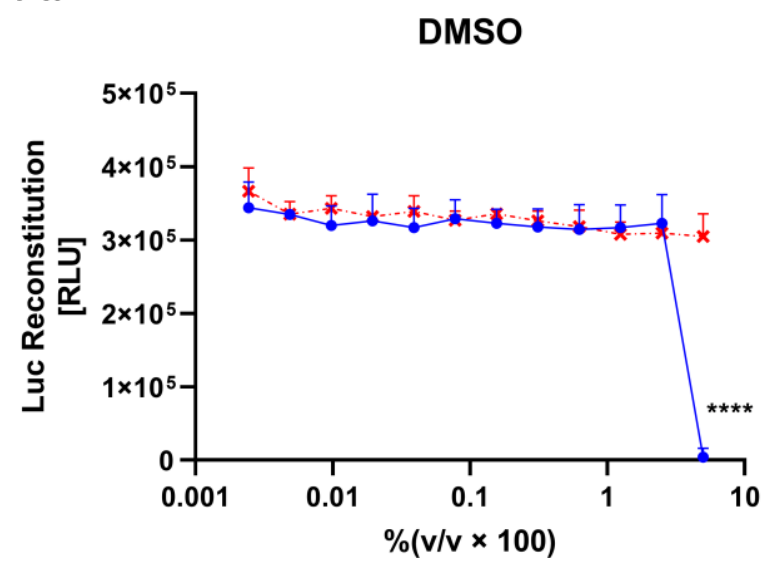

B.

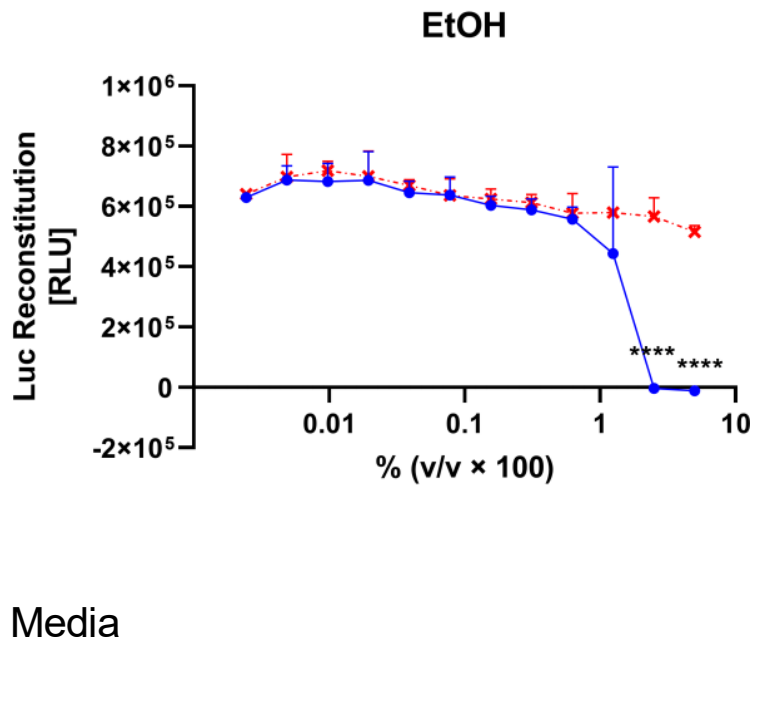

HEK 293T G8G8 cells were treated with a 2-fold dilution series of DMSO (A) or ethanol (B) in media from $5 \%$ to $0.002 \% \mathrm{v} / \mathrm{v}$. No increase in luminescence is detected due to either of these solvent components, which were used in formulating DOTAP (at $100 \mathrm{mg} / \mathrm{mL}$ ) or Lipofectamine 2000 (at proprietary concentration). Toxicity is observed for 5\% DMSO and 5\% and 2.5\% ethanol. Error bars represent standard deviation. 
Figure S8. Lipofectamine 2000 timecourse of G8G8 split-luciferase signal.

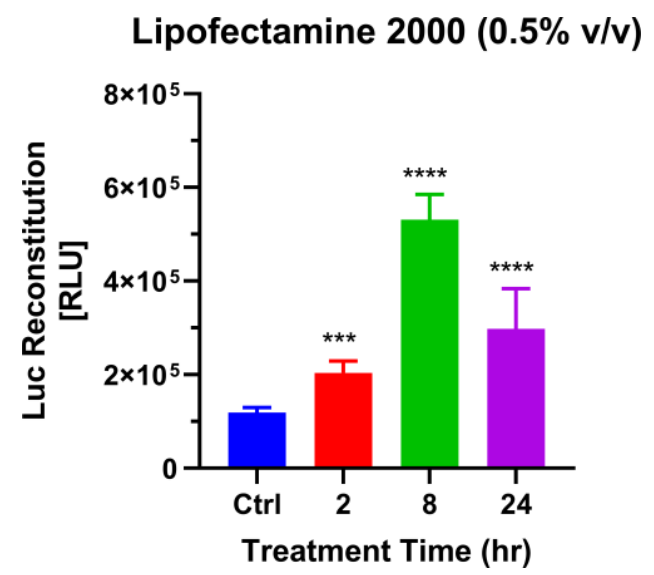

G8G8 HEK 293T cells were treated with $0.5 \%$ Lipofectamine 2000 for 2, 8, or $24 \mathrm{~h}$ and analyzed for luciferase reconstitution. Significant increases in luminescence relative to control were observed for all timepoints, with maximal response at $8 \mathrm{~h}$ post treatment. 
Figure S9. Timepoint testing of PPAA with G8G8 assay system.
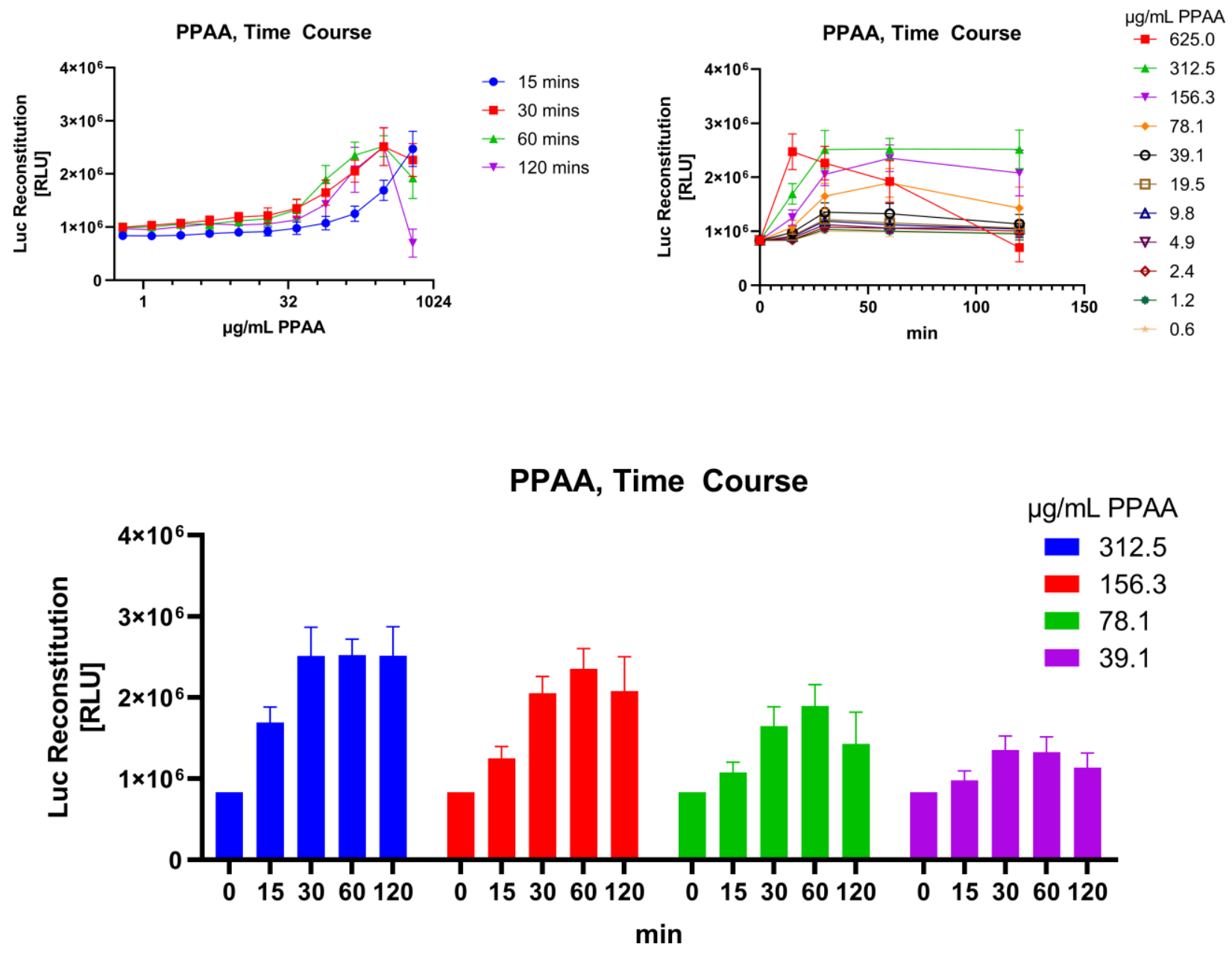

HEK 293T G8G8 cells were treated with a 2-fold dilution series of PPAA, starting at $1.25 \mathrm{mg} / \mathrm{mL}$ with treatments staggered to provide different contact times. The data from this experiment are plotted three ways. On the top left graph, the dose series for each time point is plotted.

On the top right graph, individual doses are plotted as time course series.

On the bottom graph, kinetic data for four nontoxic doses are plotted as bar charts.

Toxicity is observed at $625 \mu \mathrm{g} / \mathrm{mL}$ at $2 \mathrm{~h}$. These data reveal that endosomal disruption occurs rapidly within 15 minutes for the highest dose, but this dose causes toxicity by 2 hours. More moderate doses show robust endosome disruption without substantial toxicity within the timeframe observed. 
Figure S10. G8C2 Plasmid Map

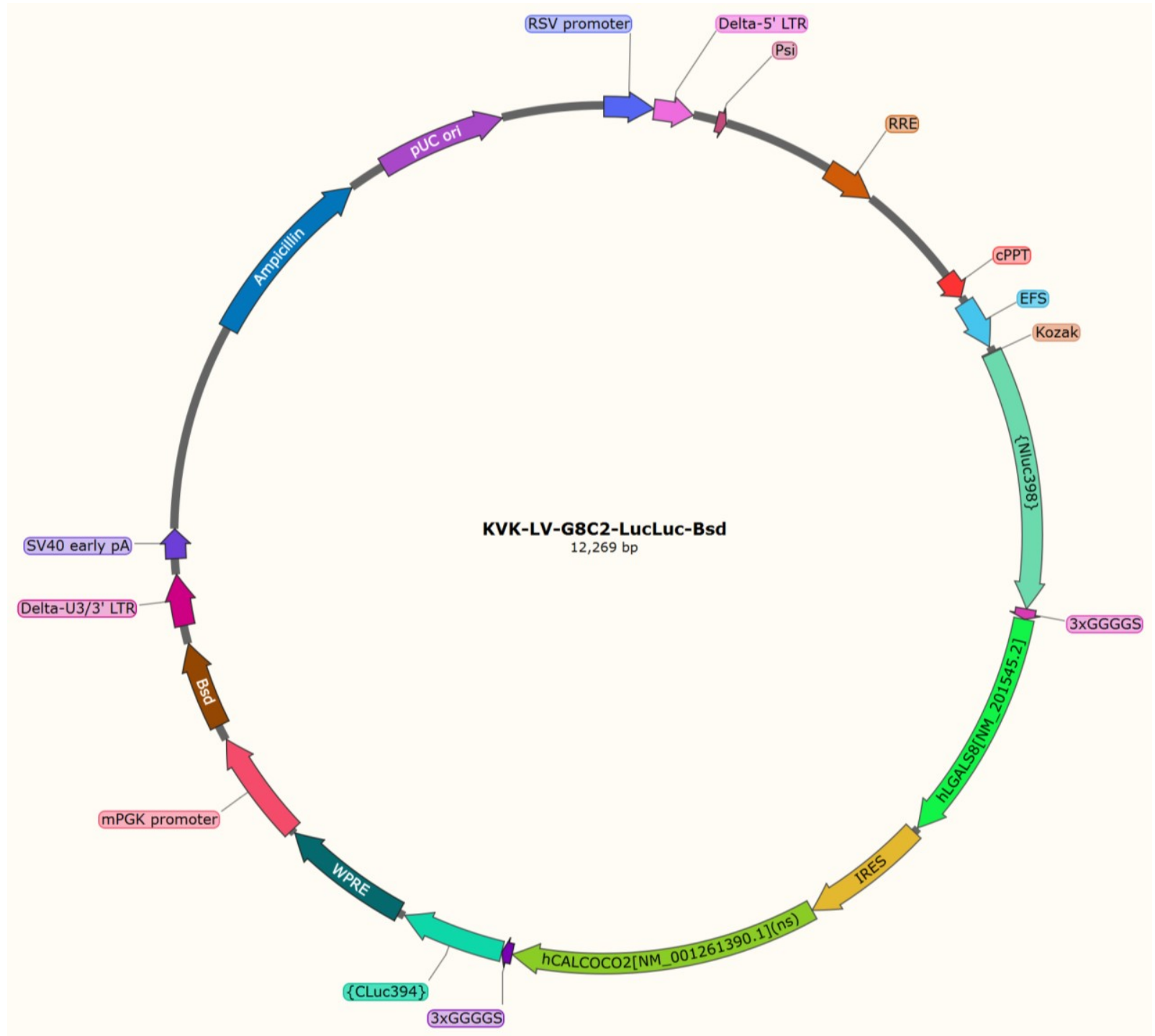

Note: DNA feature colors do not correspond to protein features in TOC graphic, Figure 1, or Figure S1. hLGALS8[NM_201545.2] is full-length Gal8. hCALCOCO2[NM_001261390.1] represents CALCOCO2.

Plasmid and full DNA sequence are available from AddGene at https://www.addgene.org/128387/ 
Figure S11. G8G8 Plasmid Map

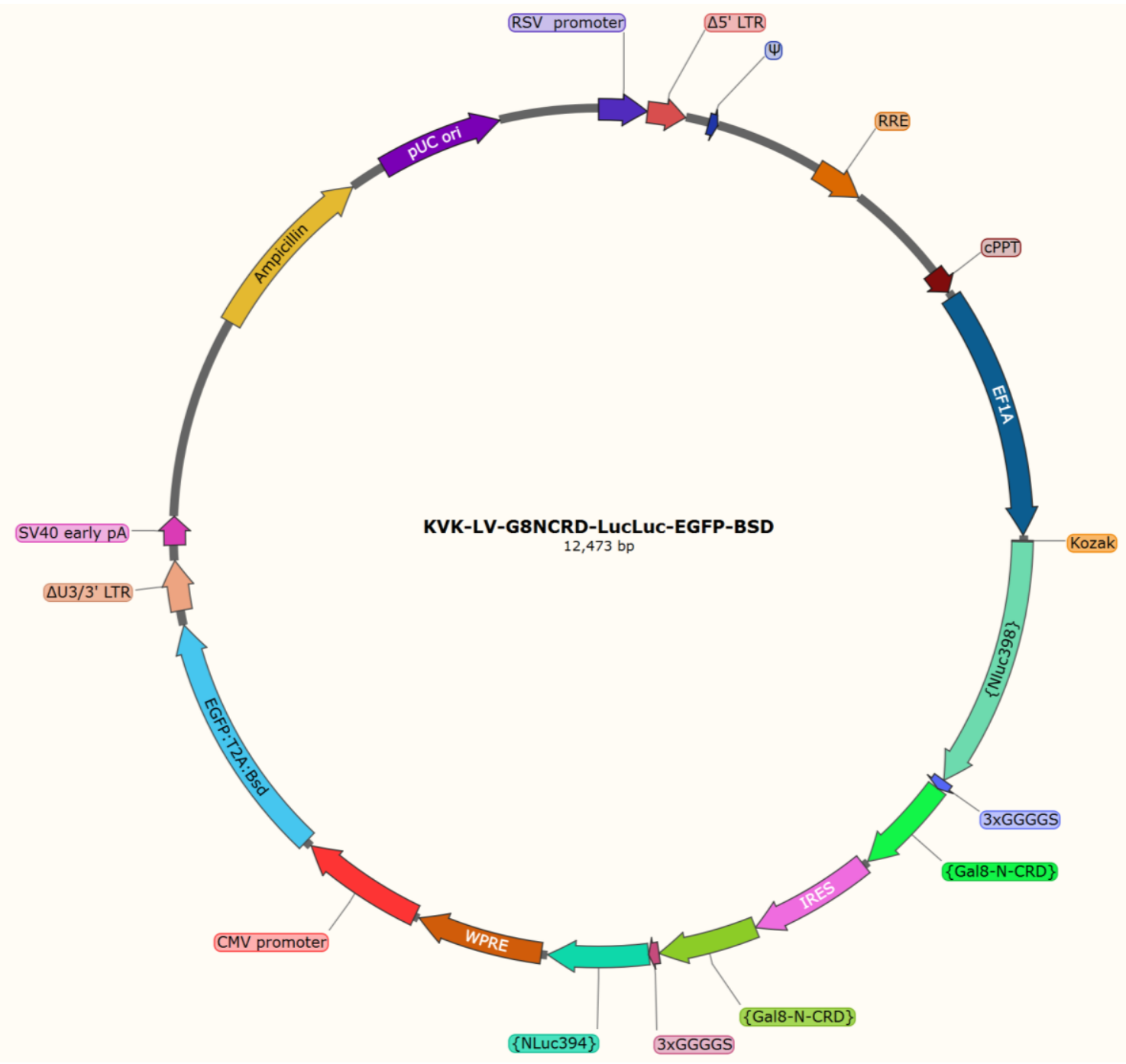

Note: DNA feature colors do not correspond to protein features in TOC graphic, Figure 1, or Figure S1. G8NCRD is referred to as "Gal8-N-CRD" in the plasmid map.

Plasmid and full DNA sequence are available from AddGene at https:/www.addgene.org/128386/ 